\title{
In vitro Remote Aspiration Embolectomy for the Treatment of Acute Ischemic Stroke
}

\author{
Asim Rizvi $^{a} \quad$ Sean T. Fitzgerald ${ }^{a}$ Kent D. Carlson ${ }^{b}$ \\ Dan Dragomir Daescu ${ }^{b}$ Waleed Brinjikji ${ }^{a}{ }^{c}$ Ramanathan Kadirvel ${ }^{a}$ \\ David F. Kallmes ${ }^{a}$ \\ a Department of Radiology, Mayo Clinic, Rochester, MN, USA; b Department of Biomedical \\ Engineering, Mayo Clinic, Rochester, MN, USA; ' Joint Department of Medical Imaging, \\ Toronto Western Hospital, Toronto, ON, Canada
}

\section{Keywords}

Acute stroke $\cdot$ Ischemic stroke $\cdot$ Remote aspiration embolectomy · Revascularization ·

Thrombolysis in Cerebral Infarction

\begin{abstract}
Background: "Remote aspiration," using suction from the proximal internal carotid artery (ICA) to open terminus occlusions, has been reported in small case series. However, it remains unclear whether remote aspiration is feasible for middle cerebral artery occlusions in the setting of potential inflow from communicating arteries. We performed an in vitro study to assess whether suction applied at various locations proximal to an occlusion could successfully aspirate the clot. Methods: A glass model of $4 \mathrm{~mm}$ inner diameter (ID) with $1 \mathrm{~mm}$ distal narrowing and $2 \mathrm{~mm}$ side branch to simulate a communicating artery was constructed. A proximal side branch was placed to simulate inflow from the proximal ICA. The impact of three different-sized catheters (ID 0.088, 0.070, and 0.056 in) on histologically different (red blood cell-cell rich, fibrin-rich, and mixed) clot analogues was tested with the catheter tip placed remotely either distal or proximal to the collateral branch. Aspiration was attempted with (1) open system (flow in both the ICA and the collateral branch, (2) flow arrest with open collateral (no flow in the ICA, but flow in the collateral branch), and (3) closed system (no flow in either the ICA or the collateral branch). The outcome was success or failure of remote aspiration. Results: For the 0.088 -in catheter, remote aspiration was successful in all conditions. For the 0.070 -in catheter, remote aspiration was unsuccessful without proximal flow arrest, but was successful in all other scenarios. For the 0.056 -in catheter, remote aspiration was success-
\end{abstract}


ful only with complete flow arrest. Conclusions: In a noncollapsible system, remote aspiration can be successfully achieved even in the setting of prominent branch arteries by using relatively large aspiration catheters. Proximal flow arrest may facilitate successful remote aspiration for some catheter sizes.

(c) 2018 S. Karger AG, Basel

\section{Introduction}

In the recent past, numerous techniques and devices have been proposed for use in urgent embolectomy for the treatment of patients with acute ischemic stroke secondary to emergent large vessel occlusion. The vast majority of these techniques and devices mandate direct contact of the embolus with the embolectomy device. Recent case series have demonstrated that in the setting of internal carotid artery (ICA) terminus occlusion, aspiration via a proximal guiding catheter alone may result in successful removal of the embolus [1]. This latter technique is termed remote, noncontact, or "no-touch" aspiration, in contrast to the usual contact aspiration performed with current distal access catheters.

Contact aspiration has important potential shortcomings primarily related to the risk for downstream embolization. These emboli may result from direct manipulation of the clot, clot ingestion that occurs distal to communicating arteries, or corked clots pulled into guide catheters without flow arrest. Accordingly, complete revascularization rates with Thrombolysis in Cerebral Infarction (TICI)-3 flow are lower at approximately 50-60\% with the most modern approaches of endovascular embolectomy. This pertains to the fact that the majority of resultant distal branch occlusions due to distal embolization subsequently make the difference between TICI-2B and TICI-3 during the endovascular procedure [2, 3].

The current study therefore sought to optimize clinical outcomes utilizing in vitro remote aspiration using catheters that are positioned proximal to communicating arteries, and with use of proximal flow arrest, which may result in full clot removal and mitigation of the risk for distal embolization.

\section{Methods}

\section{Creation of Clot Phenotypes}

Rabbit blood was obtained and experimental clot analogues with different histological proportions of red blood cells (RBCs) and fibrin were created, including RBC-rich, fibrin-rich, and mixed clots. The technique used to create and quantify the composition of the clots has been described previously [4]. Three different clot analogue types were created, with three clot replicates of $2 \mathrm{~cm}$ for each type in 1-mL syringes. All clots were formed in a static environment.

\section{Histological Confirmation}

Histological confirmation of these clot analogues was subsequently performed as follows: (1) RBC-rich clots containing 90\% RBCs, 7\% fibrin, and 3\% white blood cells (WBCs), (2) fibrin-rich clots containing 97\% fibrin, 2\% RBCs, and 1\% WBCs, and (3) mixed clots containing 35\% RBCs, 64\% fibrin, and 1\% WBCs.

Cerebrovascular Flow Loop Model

In vitro testing for remote embolus aspiration was subsequently performed utilizing the cerebrovascular flow loop design. The $4 \mathrm{~mm}$ inner diameter (ID) glass-made flow loop was designed with $2 \mathrm{~mm}$ ID for the ICA and collateral branches, and a $1 \mathrm{~mm}$ ID for downstream pinch-point constriction, simulating a middle cerebral artery occlusion for clot lodgment (Fig. 1-3). A mean arterial pressure of $90 \mathrm{~mm} \mathrm{Hg}$ was simulated for the flow loop model by positioning the fluid reservoir, filled with distilled water, $122 \mathrm{~cm}$ above the model. Two-way valves were used at the proximal and distal branch openings, simulating ICA and collateral branch 


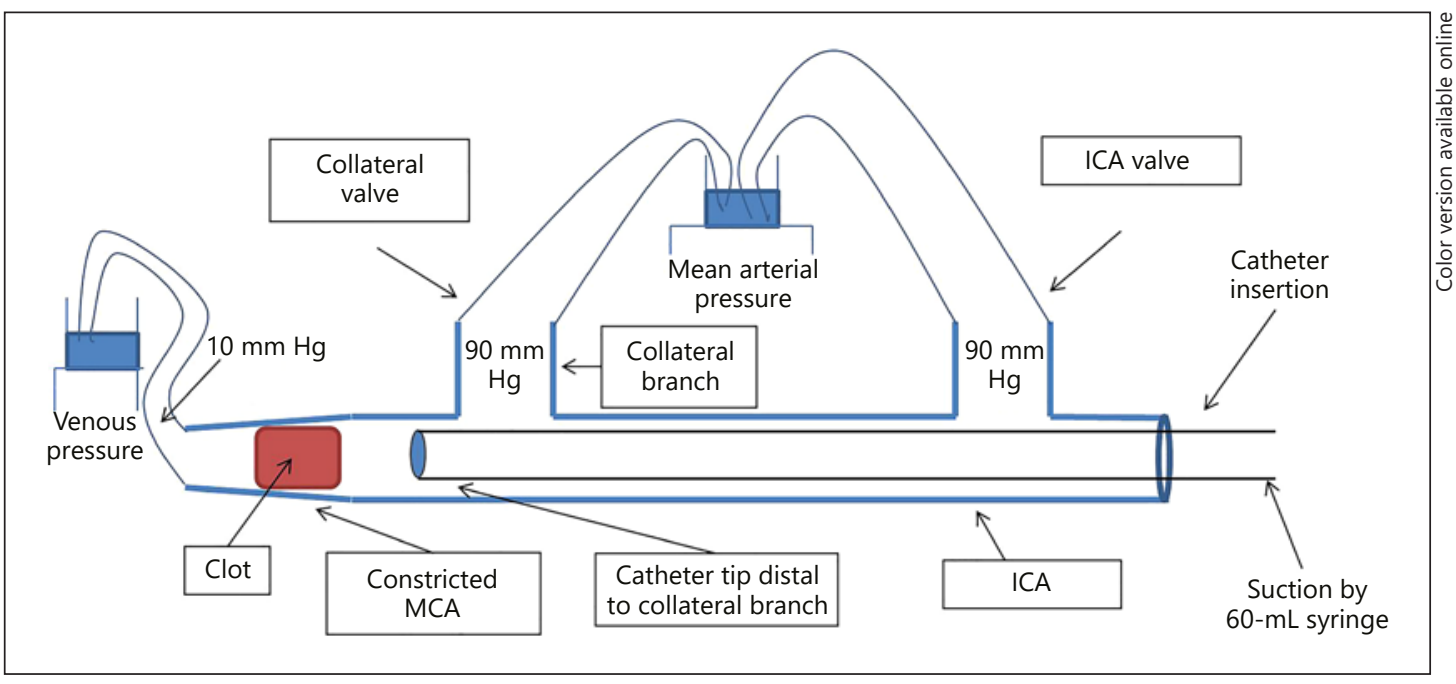

Fig. 1. Cerebrovascular flow loop model. ICA, internal carotid artery; MCA, middle cerebral artery.

in the glass model, to control fluid flow (Fig. 1-3). The different clot analogues, created in 1-mL syringes, were directly introduced through a cut-end of the syringe via opening at the proximal end of the glass model. The valve at the proximal branch opening was then opened to allow fluid flow to move the clot to the tapered end of the glass model. To minimize variability in clot lodgment at the pinch-point constriction of the model and subsequent force needed for clot retrieval, all experiments were carried out using the same technique for different clot analogue types.

\section{Aspiration Catheters}

Three different-sized catheters were used: 8-Fr 0.088-in (Cordis, Vista Brite Tip), 6-Fr 0.070-in (Cordis, Envoy), and 5-Fr 0.056-in (Cordis, Envoy) guiding catheters. The impact of these different-sized catheters was tested for various remote embolectomy scenarios in the glass model with the tip of the catheter placed either $1 \mathrm{~cm}$ distal or $1 \mathrm{~cm}$ proximal to the collateral branch. Additionally, the "ingestion" or "corking" of clots at the catheter tip was also assessed. By extension, with "clot ingestion," the aspiration flow rate draws the clot into the catheter lumen and into the syringe, while with "clot corking," the clot is rather latched or corked onto the catheter tip followed by withdrawal of the catheter under aspiration.

\section{In vitro Remote Aspiration}

Remote aspiration using each aforementioned catheter was tested for RBC-rich, fibrin-rich, and mixed clots under three different scenarios: (1) open system (both valves open, with flow in both the ICA and the collateral branch), (2) flow arrest with open collateral (proximal valve closed and distal valve open, with no flow in the ICA to simulate proximal flow arrest, but flow in the collateral branch), and (3) closed system (both valves closed, with no flow in either the ICA or the collateral branch). To achieve remote aspiration under these three different scenarios, vigorous suction was performed using a 60 -mL syringe by pulling the plunger at once to attain maximum vacuum in a homogeneous fashion. The outcome was success or failure of in vitro remote aspiration embolectomy. All scenarios were repeated three times to confirm the reliability of findings.

\section{Results}

The study findings are based on three different-sized catheters, catheter tip location, and three experimental clot analogues under three different scenarios including open system, flow arrest with open collateral, and closed system. All scenarios were tested three times with similar findings. 
Fig. 2. In vitro flow loop model

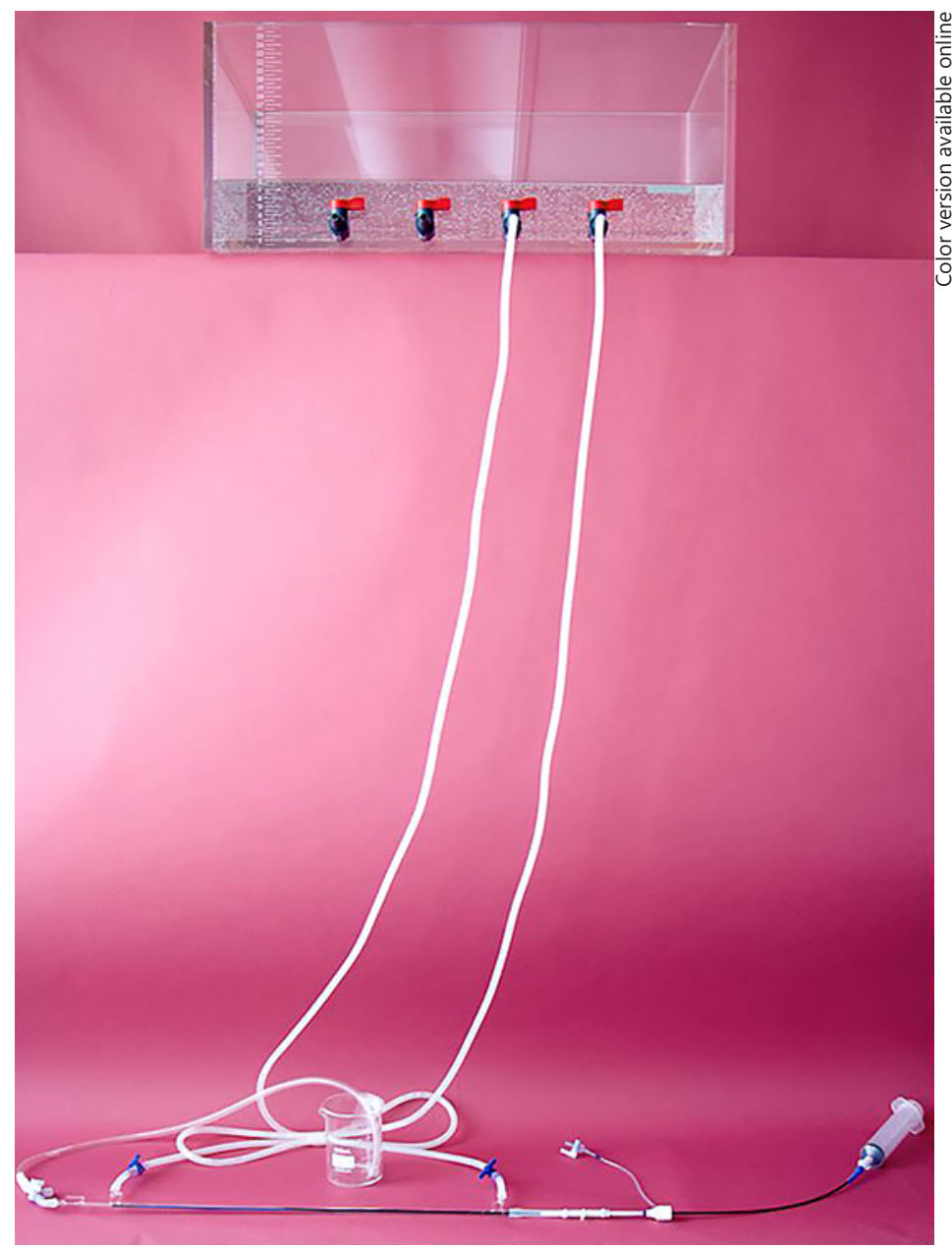
setup.

8-Fr 0.088-in Catheter (Cordis, Vista Brite Tip)

Using a large-bore 8-Fr 0.088-in catheter, remote aspiration was successful in achieving revascularization for all clot types (RBC-rich, fibrin-rich, and mixed) under open system, flow arrest with open collateral, and closed system, with the catheter tip placed either distal or proximal to the collateral (Fig. 4).

\section{6-Fr 0.070-in Catheter (Cordis, Envoy)}

Using a 6-Fr 0.070-in catheter, remote aspiration was successful in achieving revascularization for all clot types using the closed system as well as with flow arrest and open collateral, with the catheter tip placed either distal or proximal to the collateral. However, remote aspiration was not successful in the open system (Fig. 4).

\section{5-Fr 0.056-in Catheter (Cordis, Envoy)}

Using a 5-Fr 0.056-in catheter, remote aspiration was successful in achieving revascularization for all clot types using the closed system only, with the catheter tip placed either distal or proximal to the collateral. However, remote aspiration was not successful in the open system or with flow arrest and open collateral (Fig. 4). 


\section{Interventional Neurology}
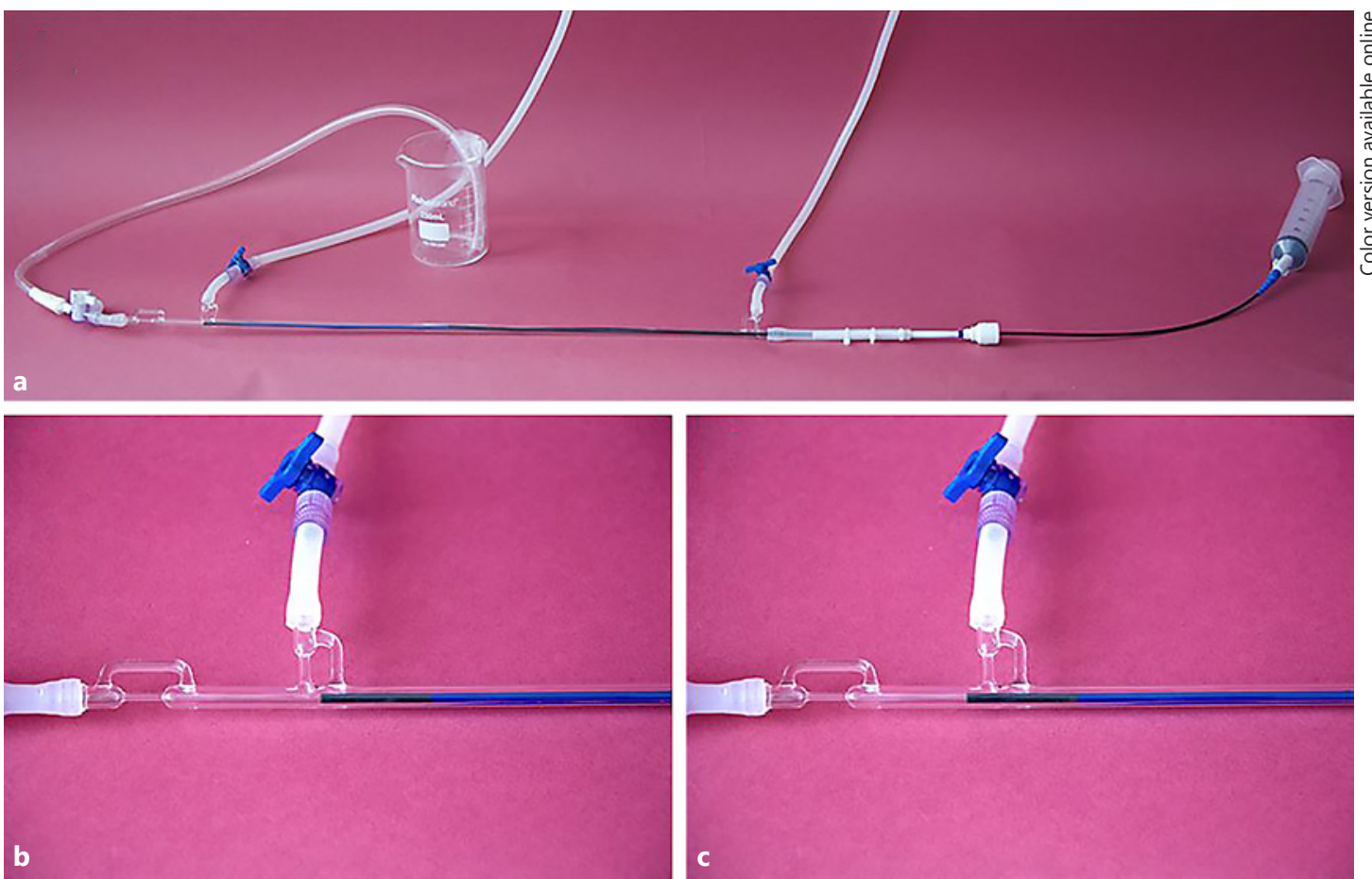

Fig. 3. a Glass-made flow loop model. b, $\mathbf{c}$ Different scenarios for in vitro remote aspiration with catheter tip proximal to collateral (b) and catheter tip distal to collateral (c).

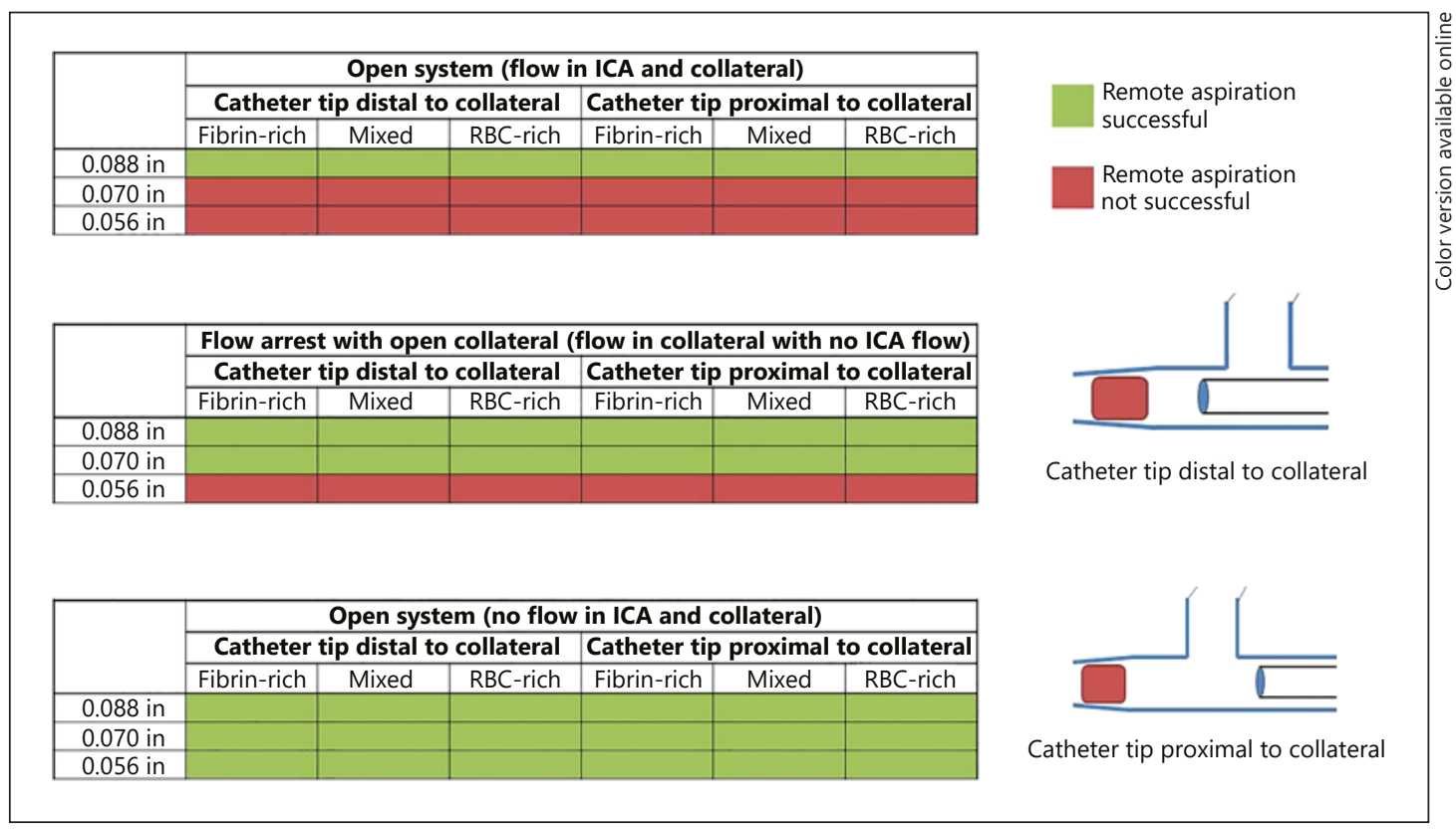

Fig. 4. In vitro remote aspiration embolectomy. ICA, internal carotid artery; RBC, red blood cell. 


\section{All Catheter Types}

Most notably, "ingestion" of the clots, and not "corking," was observed at the catheter tip in all in vitro remote aspiration experiments, regardless of catheter size, catheter tip location, and histologically different clot phenotypes.

\section{Discussion}

Our in vitro study findings reveal that remote aspiration, even in the setting of inflow from intervening communicating arteries, can be successfully achieved with large-bore aspiration catheters. Using catheter sizes similar to those of currently used distal access catheters, remote aspiration was successfully achieved, but only in the setting of proximal flow arrest. Nonetheless, very large catheters, larger than current distal access catheters, achieved revascularization remotely even without proximal flow arrest. It is likely that high flow rates through large-bore catheters lead to diminished intraluminal pressure that is transmitted to the face of the clot. Since flow is proportional to the lumen diameter to the fourth power, even modest changes in lumen size yield markedly increased flow rates.

Remote aspiration represents an attractive alternative to current techniques because it does not require direct manipulation of the clot. Previous investigators have shown that distal emboli may be induced with any manipulation (i.e., crossing the lesion with wire and catheter, advancing large-bore aspiration catheter along the clot, etc.) $[5,6]$. Moreover, positioning of the aspiration catheter tip proximal to communicating arteries, in conjunction with proximal flow arrest, would allow clot removal with minimal risk of any debris traveling downstream. Taken together, these findings suggest that remote aspiration may offer a safe, effective mode for embolectomy.

In the recent past, investigators have shown that ICA terminus occlusions may be successfully opened with suction from a proximal guide catheter in the setting of an occlusion balloon [1]. Notably, full recanalization was seen in those small case series, adding evidence to the notion that noncontact aspiration in the setting of flow arrest yields optimal angiographic outcomes. Those previous case series, however, are fundamentally different from the in vitro model tested here, as there are no substantial intervening communicating arteries between the ICA terminus and the proximal ICA. In these cases, assuming a noncollapsible vessel, the negative pressure is efficiently transmitted to the face of the clot to facilitate removal. Our study adds important additional information, since it suggests that remote aspiration may be feasible with large enough catheters even in the setting of a middle cerebral artery occlusion with patent communicating arteries.

To this end, other previous investigators, using a computational modeling approach, concluded that noncontact aspiration would be highly unlikely to generate sufficient negative pressure to achieve clot removal [7]. We note that those investigators did not model proximal flow arrest, and also that they used very-small-diameter model catheter systems. Thus, their computational setup cannot be directly compared to our own empiric data collection system.

Our study suffers a number of relevant limitations. We used noncollapsible walls without patient-specific geometries and a material (glass) which does not necessarily reflect the characteristics of human intracranial vasculature. In real clinical practice, the ICA can collapse by forced negative pressure, especially in the cervical portion. However, our study is simply a "proof of concept" experiment that suffers from lack of collapsible vessels. Future in vitro studies could readily include more realistic material such as endothelial cell-lined bioreactors using realistic, patient-specific anatomies and pulsatile flow conditions. Additionally, we surmise that collapsible vessels between the catheter tip and clot would negate the efficacy of remote aspiration, but the degree and extent of collapse of the human intracranial vessels remains poorly studied. We used a static flow system, and without forward flow we may have 
overestimated the efficacy of remote aspiration. We used distilled water instead of blood as our fluid, which also may have impacted the results. The clots used were substantially more homogeneous than those encountered during a standard embolectomy procedure. Moreover, the lodgment of clots at the tapered end of the glass model may have resulted in some variability in terms of how "stuck" the clots were and may have subsequently impacted the results with regards to force needed for clot retrieval. The static coefficient of friction in our model between the clot and glass tube is likely substantially different from that of a clot and a vessel wall. Lastly, we modeled only a single diameter of communicating branch artery, and almost certainly results will be impacted by the rate of inflow from varying diameters of these vessels.

Notwithstanding the important limitations listed above, the current study provides a foundation for future studies of remote aspiration. While recent clinical case series of remote aspiration are limited to ICA terminus occlusions, larger distal access catheter utilization may allow us to achieve remote aspiration in the setting of prominent communicating arteries.

\section{Conclusions}

Our experiments suggest that, in a noncollapsible system, remote aspiration embolectomy can be successfully achieved even in the setting of prominent branch arteries using relatively large aspiration catheters. Proximal flow arrest may facilitate successful remote aspiration for some catheter sizes.

\section{Acknowledgments}

A. Rizvi, W. Brinjikji, R. Kadirvel, and D.F. Kallmes made substantial contributions to the conception and design of the work, to the acquisition, analysis, and interpretation of data for the work, to the drafting of the work, and to revising it critically for important intellectual content. A. Rizvi and S.T. Fitzgerald made substantial contributions for data collection. K.D. Carlson and D.D. Daescu made substantial contributions to the design of the flow loop model. All authors provided final approval of the version to be published. All authors agree to be accountable for all aspects of the work.

\section{Disclosure Statement}

None of the authors reported any conflicts of interest. This research received no specific grant from any funding agency in the public, commercial, or not-for-profit sectors.

\section{References}

1 Haussen DC, Bouslama M, Grossberg JA, Nogueira RG. Remote aspiration thrombectomy in large vessel acute ischemic stroke. J Neurointerv Surg. 2017 Mar;9(3):250-2.

2 Gratz PP, Schroth G, Gralla J, Mattle HP, Fischer U, Jung S, et al. Whole-brain susceptibility-weighted thrombus imaging in stroke: fragmented thrombi predict worse outcome. AJNR Am J Neuroradiol. 2015 Jul;36(7):127782.

3 Klinger-Gratz PP, Schroth G, Gralla J, Jung S, Weisstanner C, Verma RK, et al. Protected stent retriever thrombectomy prevents iatrogenic emboli in new vascular territories. Neuroradiology. 2015 0ct;57(10):1045-54.

4 Duffy S, Farrell M, McArdle K, Thornton J, Vale D, Rainsford E, et al. Novel methodology to replicate clot analogs with diverse composition in acute ischemic stroke. J Neurointerv Surg. 2017 May; 9(5):486-91.

5 Chueh JY, Kühn AL, Puri AS, Wilson SD, Wakhloo AK, Gounis MJ. Reduction in distal emboli with proximal flow control during mechanical thrombectomy: a quantitative in vitro study. Stroke. 2013 May;44(5):1396-401.

6 Chueh JY, Puri AS, Wakhloo AK, Gounis MJ. Risk of distal embolization with stent retriever thrombectomy and ADAPT. J Neurointerv Surg. 2016 Feb;8(2):197-202.

7 Lally F, Soorani M, Woo T, Nayak S, Jadun C, Yang Y, et al. In vitro experiments of cerebral blood flow during aspiration thrombectomy: potential effects on cerebral perfusion pressure and collateral flow. J Neurointerv Surg. 2016 Sep;8(9):969-72. 